\title{
Review
}

\section{Monte Carlo radiation transport in external beam radiotherapy}

\author{
Yiğit Çeçen \\ a Department of Radiation Oncology, Akdeniz University, 07058 - Antalya, Turkey \\ *Corresponding author: ycecen@akdeniz.edu.tr
}

\begin{abstract}
The use of Monte Carlo in radiation transport is an effective way to predict absorbed dose distributions. Monte Carlo modeling has contributed to a better understanding of photon and electron transport by radiotherapy physicists. The aim of this review is to introduce Monte Carlo as a powerful radiation transport tool. In this review, photon and electron transport algorithms for Monte Carlo techniques are investigated and a clinical linear accelerator model is studied for external beam radiotherapy. The statistical uncertainties and variance reduction techniques for Monte Carlo simulation are also discussed.
\end{abstract}

\section{Keywords: Electron transport, Monte Carlo, photon transport, radiotherapy}

\section{Introduction}

Radiotherapy is the clinical process where ionizing radiation is used for the treatment of cancer. Approximately $60 \%$ of all cancer patients receive radiotherapy as a component of their treatment. The aim of radiotherapy is to deliver a prescribed radiation dose to a well-defined tumor volume with minimal damage possible to the surrounding healthy tissue (Perez 2008).

The delivered dose is the main concern of radiotherapy physicists. Since the therapeutic doses for cancer treatment is high, dose calculation has the upmost importance in radiotherapy. Delivering a decreased dose than planned will reduce the probability of tumor control; on the contrary, delivering a higher dose may cause acute or chronic side-effects, even death. The ICRU recommends $\pm 5 \%$ accuracy for dose-delivery computations (ICRU-50 1993). Today, computerized treatment planning systems (TPS) calculate dose distributions using several different algorithms. The dose algorithm is the most unique, critical, and complex piece of software in a computerized planning system (Van Dyk 1999). With respect to the conventional algorithms with approximations, Monte Carlo technique is introduced as a 'gold standard' for photon dose calculations which aim to simulate the complete microscopic radiation transport in the patient (Scholz 2003). Kernel based algorithms like pencil beam kernel, cone convolution, and superposition can calculate almost all dose distributions within $\pm 5 \%$. These TPS algorithms work well with little density changes in the target path. The brain tumor is an example of little density changes with only the skull in the path which creates a density difference. However, many favor Monte Carlo simulation in radiotherapy for use in high inhomogeneity gradients like head \& neck regions. (Chaves 2001; Leal 2003; Schwarz 2003).

\subsection{What is Monte Carlo?}

Monte Carlo is a numerical method for simulating the behavior of various physical and mathematical systems. It provides a solution to a problem that model objects interacting with other objects or with their environment based on simple relationships (Bielajew 2001). The origin of the name Monte Carlo comes from its gambling aspect. Every Monte Carlo simulation is based on randomly happening events so that the outcome is not always absolute.

The general idea of Monte Carlo analysis is simulation. The idea is to build a model which, in all means, is able to represent the real system of interest. Interaction probabilities (of any kind) must be well defined within the system by probability distribution functions (PDF) (Figure 1).

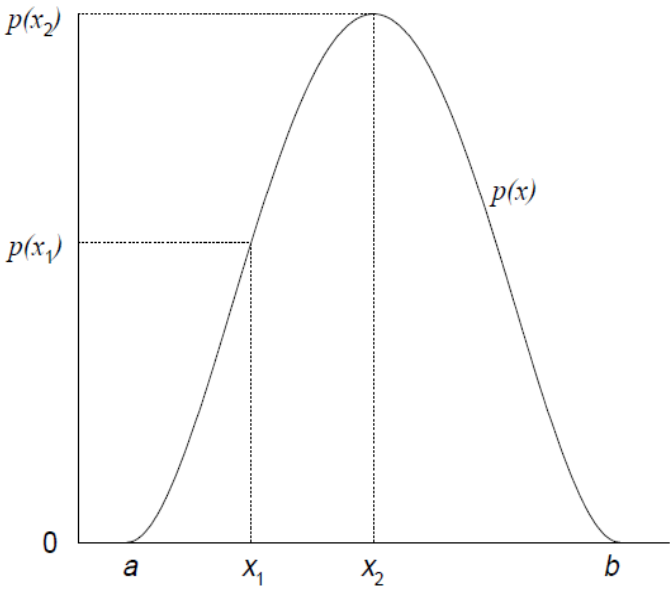

Figure 1. A typical probability distribution function.

$$
P D F=\int_{a}^{b} p(x) d x=1
$$

These PDFs describe the behavior of a large number of events. The behavior of the entire system is inferred from the average behavior of the simulated events, using the central limit theorem, which states that, as the number of trials approaches infinity, the average result of the sample approaches the true solution. This is in contrast to deterministic methods, which explicitly solve the 
equation. The trials are generated by inserting a random number into the probability distribution function. These random numbers are randomly generated variables which are uniformly distributed between 0 and 1 . In Monte Carlo simulation, computer based random numbers (pseudo random numbers) are used as random number generators (RNG).

Today's applications of Monte Carlo Method include reactor design, radiation therapy, traffic flow, stock exchange predictions, weather forecasting, behaviors of nanostructures and polymers, oil-well exploration and so on (Zaidi 2003).

\section{Monte Carlo for Radiotherapy}

The application of Monte Carlo modeling in radiation therapy is a growing, enthusiastic topic which has unexplored ends. An accurate dose distribution calculation is a requirement of radiotherapy and several other fields like nuclear medicine imaging, radionuclide therapy, and radioprotection. In radiation therapy applications, the system is the transport of radiation particles (i.e. photons and electrons) through a defined geometry, and the events are interactions in matter. The probabilities of possible interactions of radiation in matter are well known, and depend on the particle's energy and material it travels in. Random number selection dictates a particle interaction based on PDFs and a large number of trials will produce a true distribution of events.

\section{1. Photon Transport}

In photon transport, the major photon interactions with matter include the photoelectric effect, Compton scattering, pair-production, Rayleigh scattering, and photonuclear interactions. These interactions are governed with photon cross sections of the travelled medium. Photon cross sections refer to the various kinds of attenuation coefficients. They play an essential role in particle transport simulations. The probability of occurrence of a certain kind of interaction depends on its relative contribution to the total cross section. The probability of photon interaction can be expressed in terms of the linear attenuation coefficient $\mu$, which indicates the fraction of incident photons that will interact per unit thickness of the attenuating medium.

$$
\mu=\sigma_{\text {photoelectric }}+\sigma_{\text {compton }}+\sigma_{\text {pair_production }}+\sigma_{\text {rayleigh }}
$$

where

$$
\sigma_{\text {photoelectric }}, \sigma_{\text {compton }}, \sigma_{\text {pair_production and }} \sigma_{\text {rayleigh }}
$$

are cross sections for photoelectric effect, Compton scattering, pair production and Rayleigh scattering respectively. The contribution from photonuclear interactions is often excluded. The inverse of $\mu$ is the mean free path $\lambda$, in units of length.

$$
\lambda=\frac{1}{\mu}
$$

It indicates the average distance traveled by a particle before undergoing an interaction. The exponential nature of photon attenuation can be represented by:

$$
N=N_{\mathrm{o}} e^{-\mu x}
$$

where $N$ is the number of photons transmitted through a medium of thickness $x$ with $N_{o}$ incident photons. Since $\mu$ depends on the material density, it is often scaled by the mass, atomic, or electron density.

Typically, the mass attenuation coefficient $\mu_{m}$ is in units of $\mathrm{cm}^{2} / \mathrm{g}$, the atomic attenuation coefficient $\mu_{a}$ is in units of $\mathrm{cm}^{2} /$ atom, and the electronic attenuation coefficient $\mu_{e}$ is in units of $\mathrm{cm}^{2} /$ electron. These quantities are related as following:

$$
\mu=\rho \mu_{m}=\frac{\rho N_{A}}{A} \mu_{a}=\frac{\rho N_{A} Z}{A} \mu_{e}
$$

where $N_{a}$ is the Avogadro's number, $\mathrm{Z}$ is the atomic number, and $A$ is the mass number of the attenuator. Photon interaction cross sections depend on the photon energy and the atomic number of the medium. As an illustrative example, Figure 2 shows the fraction of the total cross section for each interaction for carbon and tungsten. The data is obtained from the NISTXCOM database. Carbon represents low-Z materials whereas tungsten represents high-Z materials.

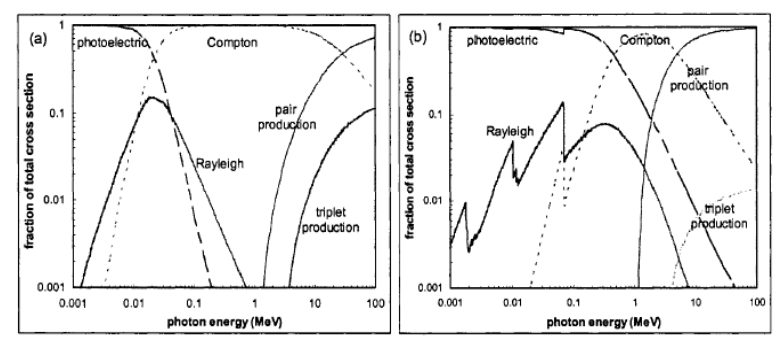

Figure 2. Contribution of each photon interaction type to the total cross section for (a) carbon and (b) tungsten. (NISTXCOM database)

In Monte Carlo simulation, particles are transported on a history by history basis. A history batch contains all the information of particles and their secondary created particles in the geometry of interest until the energy of each particle falls below a cut-off value. This procedure increases efficiency by depositing the energy of the photon locally when it is too small to be of significance, rather than tracking its full trajectories until it gets absorbed.

In general, the distance to the next interaction, $s$, can be sampled from the total cross section $\mu$, in units of $\mathrm{cm}^{-1}$ :

$$
s=\frac{1}{\mu} \ln R_{1}
$$

where $R_{1}$ is a random number uniformly distributed between 0 and 1 . If the photon remains in the volume of interest after it is transported, the branching ratios of the photon interactions will be sampled to determine which interaction will follow. The sampling requires another random number $R_{2}$ uniformly distributed between 0 and 1. Since the contribution of each interaction reflects its probability of occurrence, it can be stated that; photoelectric effect will happen if,

$$
0<R_{2} \leq \sigma_{\text {photoelectric }} / \mu
$$

Compton scattering will happen if,

$$
\sigma_{\text {photoelectric }} / \mu<R_{2} \leq \sigma_{\text {photoelectric }}+\sigma_{\text {compton }} / \mu
$$


pair production will happen if,

$\sigma_{\text {photoelectric }}+\sigma_{\text {compton }} / \mu<R_{2} \leq \sigma_{\text {photoelectric }}+\sigma_{\text {compton }}+\sigma_{\text {pair_production }} / \mu$

and Rayleigh scattering will happen if,

$$
\sigma_{\text {photoelectric }}+\sigma_{\text {compton }}+\sigma_{\text {pair_production }} / \mu<R_{2} \leq \mu
$$

After the interaction, the parameters of the resultant particles will be sampled. The procedure continues until every particle is processed. At that point, a new particle history can start.

\section{2. Electron Transport}

Electron interactions can be separated into two sections catastrophic events and soft events. If electrons lose their energy via soft events, there needs to be a large number of interactions on the order of 105-106 before an electron lose all of its energy. So it is sensible to condense these interactions into large step sizes (Figure 3).
Electron track

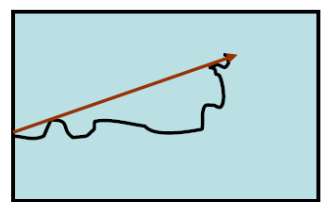

\section{Condensed track}

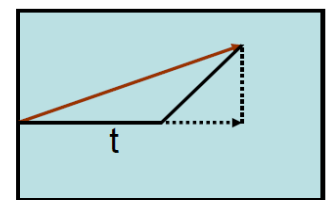

Figure 3. Condensing electron interactions into large step sizes for soft events.

These step sizes should be large enough so that sufficient number of interactions occurs within each step for energy loss models to be valid, and they should be small enough to reduce errors due to the approximation of electron tracks into line segments. The soft events can be summarized as, low-energy Møller (Bhabha) scattering (modeled as part of the collision stopping power), atomic excitation (modeled as another part of the collision stopping power), soft bremsstrahlung (modeled as radiative stopping power), and elastic electron (positron) multiple scattering from atoms. (Bielajew 2001).

However, it is relatively easy to model catastrophic events. These events can be summarized as, large energyloss Møller scattering $\left(\mathrm{e}^{-} \mathrm{e}^{-} \rightarrow \mathrm{e}^{-} \mathrm{e}^{-}\right)$, large energy-loss Bhabha scattering $\left(\mathrm{e}^{+} \mathrm{e}^{-} \rightarrow \mathrm{e}^{+} \mathrm{e}^{-}\right)$, hard bremsstrahlung emission $\left(\mathrm{e}^{ \pm} \mathrm{N} \rightarrow \mathrm{e}^{ \pm} \gamma \mathrm{N}\right)$, and positron annihilation inflight and at rest $\left(\mathrm{e}^{+} \mathrm{e}^{-} \rightarrow \gamma \gamma\right)$. Electron interactions are mainly dependent on material composition. The electron stopping powers (both collision and radiative) play an important role.

For external beam radiotherapy radiation transport, one should couple photon and electron transport algorithms because photons will produce secondary electrons and electrons will produce secondary photons. The important issue is to score and track every individual particle until the pre-determined cut-off energy.

\section{3. Source Modelling}

In radiotherapy treatment planning, most dose calculation algorithms require a commissioning process that calibrates the algorithm such that calculated results and measured data agree for standard fields. For a linear accelerator, each combination of energy and field size must also be commissioned. For kernel based analytical dose calculation algorithms, measurements are used as a starting point and the model parameters are adjusted by the use of optimization techniques such that measurements and calculated doses agree for the benchmark cases (Cecen 2008).

However, for Monte Carlo dose calculations, the resulting dose distributions are entirely defined by the radiation field, and not by any model approximation parameters. As the dose calculation is based on welldefined principles of transport physics, the entire dose calculation only depends on the accurate modeling of the medium and the radiation field.

It is important to create a realistic model of the linear accelerator's treatment head, known as the beam modeling. The beam modeling provides the energy, positional and angular distributions of photons and electrons exiting the machine, known as the phase space data (PSD). The phase space data is defined as the collection of variables that describe the particle's absolute location and direction in space with respect to the origin of the coordinate system. Phase space data also contains the type, energy and weight of the particle.

$$
\Phi\{\text { type }, E(n), \vec{x}(n), \vec{u}(n), \text { weight }(n)\}
$$

where $n$ is the history number, type is the type of radiation (electron or photon for our case), $E$ is the current energy of the particle, $\vec{x}$ is the location vector for $(x, y, z), \vec{u}_{\text {is }}$ the direction vector $\vec{u}=(u, v, w)$ or $\vec{u}=(\sin \theta \cos \phi, \sin \theta \sin \phi, \cos \theta)$ and weight is the weight of the particle. A particle weight may be included in order to improve simulation efficiency if an interaction has been forced in low density material; a weighting factor must be applied to the resultant particles and all the dose deposited by those particles, track information history may be logged. The phase space vector for each particle is then collected into a data file to be used as the source for the dose calculations.

There are two questions to be answered. First question is; "where is the particle?" This question is answered by the PSD. Second question is; "where is it going?" The answer is ray tracing, which is the definition of how particles are transported in space.

$$
\begin{gathered}
\vec{x}=\vec{x}_{0}+\vec{u}_{o} s \\
\text { where } \\
\vec{x}_{0}=\left(x_{0}, y_{0}, z_{0}\right)
\end{gathered}
$$

is the current position

$$
\vec{u}_{0}=\left(u_{0}, v_{0}, w_{0}\right)
$$

is the current direction and $s$ is the distance to travel to the new position. $s$ is dependent on the type of particle. For photon transport $s$ was defined in equation.

\section{4. Linear Accelerator modeling}

Today, most of the external beam radiation treatment is performed by linear accelerators (LINACs). LINAC is a 
high energy photon and electron generator. An electron gun generates electrons and these electrons are guided onto a target to generate photons. The LINAC head should be geometrically modeled as the travelling medium for photon and electron transport. There are some essentials for the LINAC head model. Location, size and material composition of components must be known. The components of the head are to be modeled starting from the vacuum exit window. These components are the photon target, primary collimator, scattering foils for electron beams, flattening filter for photon beams, ionization chamber, mirror, wedges, secondary collimators (X-Y jaws), multi-leaf-collimators, and applicators for electron beams. As an example, a Philips SL 25 LINAC head model is shown in Figure 4.
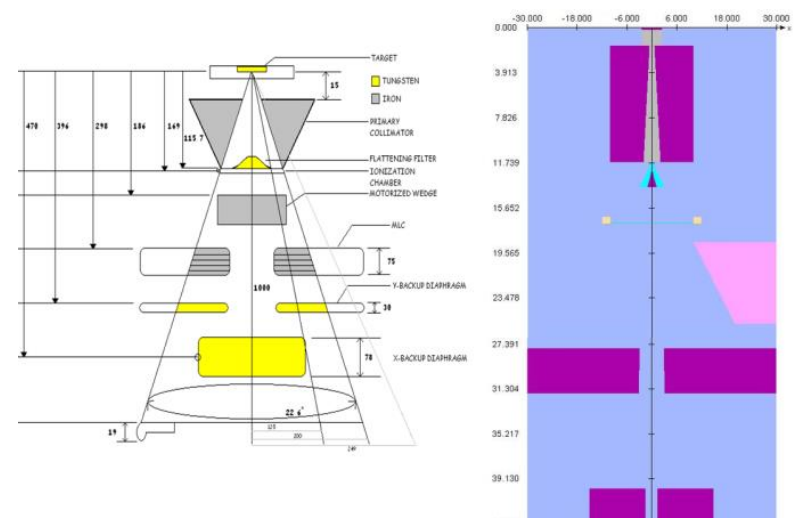

Figure 4. Philips SL 25 LINAC head model. (a) sketch of the LINAC head, (b) BEAMnrc modeled LINAC head.

The LINAC head is modeled with the help of BEAMnrc Monte Carlo code. Figure 5 shows the energy spectrum of $6 \mathrm{MV}$ photons which are generated by $6 \mathrm{MeV}$ electrons directed onto the photon target. This energy spectrum is also calculated with the help of BEAMnrc code (Cecen 2008).

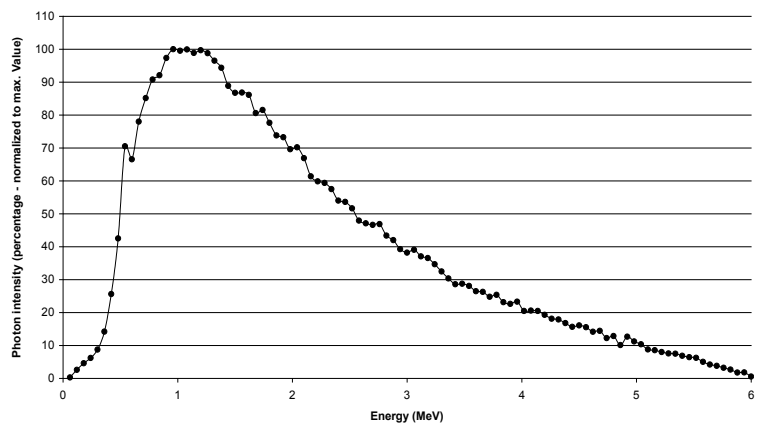

Figure 5. Energy spectrum of the Philips SL 25 LINAC for 6MV photons modeled by BEAMnrc. PSD is scored at the exit of the LINAC head.

\section{5. Dose deposition calculations}

There are two phases of Monte Carlo modeling for external beam radiation therapy. The first phase is the source modeling. The second phase is the dose calculation in the patient, or phantom geometry. Modeling of the patients (or phantoms) is to be performed on the basis of DICOM files. All information available on the TPS (angles of gantry, table, collimator, leaf and jaw settings, monitoring units (MU), and isocentre relative to CT data) comes in a DICOM RT Plan file. The CT images are carried in DICOM CT files. This file should be handled by using an interface program to convert each grey-level pixel into a corresponding anatomical tissue type defined by its elemental composition and mass density (Cecen 2008). Then the source is directed into the system to calculate for dose deposition. This is a complex model and should be handled with Monte Carlo codes.

The Monte Carlo technique for photon/electron transport is available as codes. Some of these codes are freely distributed and the reader is encouraged to investigate the following codes for use; OMEGA-BEAMnrc package, EGSnrc (Electron-Gamma-Shower), MCNP (Monte Carlo N-Particle Transport), ETRAN, ITS (Integrated Tiger Series), GEANT, PENELOPE (Penetration and Energy Loss of Positron and Electrons), DPM, FLUKA, PEREGRINE and VMC++ (Voxel Monte Carlo).

The Monte Carlo codes in literature are mainly EGS (Malataras 1996; Ayyangar 1998; Leal 2003; Reynaert 2004), MCNP (Moraleda 2004) and GEANT (Trindade 2003; Rodrigues 2004) for radiotherapy studies.

\section{Statistical uncertainties}

A direct approach of estimating uncertainties in the precision of Monte Carlo simulations is to divide the simulation of histories into $N$ equal batches, and calculate the standard error on the mean for the scored quantity $x$. The mean value;

$$
\begin{gathered}
\bar{x}=\frac{1}{N} \sum_{j=1}^{n} x_{j} \\
\text { where } \\
x_{j}=\sum_{i=1}^{n} x_{i}
\end{gathered}
$$

for the $j$ th batch

and the square of variance associated with $x$ is;

$$
s_{x}^{2}=\frac{1}{n-1} \sum_{j=1}^{n}\left(x_{j}-\bar{x}\right)^{2}=\frac{1}{n-1} \sum_{j=1}^{n}\left(x_{j}^{2}-\bar{x}^{2}\right)
$$

the square of variance associated with $\bar{x}$ is then,

$$
s_{\bar{x}}^{2}=\frac{s_{x}^{2}}{n}
$$

At the end, the result of Monte Carlo calculations is expressed as:

$$
\bar{x}=x \pm s_{\bar{x}}
$$

The number of batches should be large enough to avoid fluctuations in the uncertainties.

\section{Variance Reduction Techniques}

Variance reduction techniques are often employed in Monte Carlo simulations to increase efficiency $\varepsilon$ by selectively discarding particles that do not contribute to the scored quantities and increasing the number of particles that do. $\varepsilon$ can be defined as: 


$$
\varepsilon=\frac{1}{s_{\bar{x}}^{2} T}
$$

where

$T$ is the simulation time [CPU seconds] required to obtain an estimated variance of $s_{\bar{x}}^{2}$.

There are two ways to improve efficiency. Either one can lower the calculation time or the variance. Calculation time depends on the power of computers and better programming techniques. However there are tricks to lower the variance which are called variance reduction techniques. To reduce the relative error without increasing the number of histories, variance reduction techniques are introduced. The main goal is to reduce sample variance so that the relative error decreases. Variance reduction (biasing) techniques for Monte Carlo simulations can also reduce the amount of computer time required for obtaining results of sufficient precision. Many of the variance reduction techniques produce and/or destroy particles during the simulation per history to produce outcomes closer to the solution. In some cases, the effect of biasing the simulations to gain efficiency has to be offset by properly adjusting the weights of the scored quantities.

Variance reduction algorithms can be classified into four major categories. In the truncation methods, some aspects of the simulation that contribute little to the outcomes are truncated. Examples include not modeling the irrelevant parts of the geometry, and imposing an energy cutoff below which particles are terminated. The population control methods controls the quantities of the particles to be sampled based on their levels of importance. Methods such as particle splitting and Russian roulette belong to this category. The modified sampling methods modify the sampling distributions to improve the statistics, which may involve biasing the particle source or forcing an interaction to occur. Lastly, the partially deterministic methods incorporate some deterministic features in the sampling, which may alter the random number sequence.

\section{Conclusion}

Monte Carlo techniques have a wide range of application area in science and technology. Any complex problem can be modeled with proper probability distribution functions, a good random number generator and welldefined geometric modeling.

In radiation transport, Monte Carlo is a very precise modeling tool. Application of Monte Carlo techniques in radiation transport can be summarized as, basic dosimetry, radiation detector simulations, radiation protection and shielding calculations, brachytherapy and external beam radiotherapy. For external beam radiotherapy one can use Monte Carlo modeling for direct implementation into TPS, derivation of beam data, verification of beam models or treatment planning verification tool for IMRT and arc therapy. This study can be used for educational purposes and may provide a better understanding of Monte Carlo methods in radiotherapy.

\section{References}

Ayyangar KM, Jiang SB (1998). Do we Need Monte Carlo Treatment Planning for LINAC Based Radiosurgery? A case study. Med Dosim 23, 161-168.

Bielajew AF (2001). Fundamentals of the Monte Carlo method for neutral and charged particle transport, Ann Arbor, Michigan, The University of Michigan.

Cecen Y (2008). Monte Carlo Simulation in Radiotherapy. The Institute for Graduate Studies in Science and Engineering, PhD. Thesis, Hacettepe University, 62 pp.

Chaves A, Alves C, Fragoso M, et al. (2001). EGS4 and MCNP4b MC Simulation of a Siemens KD2 Accelerator in 6MV Photon Mode. Laboratorio De Instrumenta Cao E Fisica Experimental De Particulas.

ICRU-50 (1993). International Commission on Radiation Units and Measurements. Prescribing, Recording, and Reporting Photon Beam Therapy ICRU Report 50, Washington, DC.

Leal A, Doblado SF, Arrans R, et al. (2003). Routine IMRT Verification by Means of an Automated Monte Carlo Simulation System. Int J Radiat Oncol Biol Phys 56, 5868.

Malataras G, Kappas C, Lovelock DMJ, et al. (1996). Simulation with EGS4 Code of External Beam of Radiotherapy Apparatus with Workstation and PC Gives Similar Results? Comput Meth Prog Bio 52, 4551.

Moraleda M, Gomez-Ros JM, Lopez MA, et al (2004). A MNCP Based Calibration Method and a Voxel Phantom for in-vivo Monitoring Am-241 in Skull. Nucl Instrum \& Meth Phys Res 526, 551-559.

Perez (2008). Principles and Practice of Radiation Oncology (5 ${ }^{\text {th }}$ Edit.), Halperin EC, Perez CA, Brady LW, Wilkins LW, ISBN-13: 978-0-7817-6369-1, ISBN-10: 07817-6369-X

Reynaert N, De Smedt B, Coghe M, et al. (2004). MCDE: A New Monte Carlo Dose Engine for IMRT. Physics in Medicine and Biology.

Rodrigues P, Trindade A, Peralta L, et al. (2004). Application of GEANT4 radiation transport toolkit to dose calculations in anthropomorphic phantoms. Appl Radiat Isotop 61, 1451-1461.

Scholz C, Schulze C, Oelfke U, et al. (2003). Development and Clinical Application of a Fast Superposition Algorithm in Radiation Therapy. Radiother Oncol 69, 79-90.

Schwarz M, Bos LJ, Mijnheer BJ, et al. (2003). Importance of Accurate Dose Calculations Outside Segment Edges in IMRT Planning. Radiother Oncol 69, 305-314.

Trindade A, Rodrigues P, Peralta L, et al. (2003). Fast Electron Beam Simulation and Dose Calculation in Radiotherapy. Nucl Instrum Meth Phys Res 522, 568578.

Van Dyk J (1999). The modern technology of radiation oncology: a compendium for medical physicists and radiation oncologists, Volume 1, Medical Physics Publications, 1072 pp. ISBN: 0-9448-3838-3, 978-09448-3838-9.

Zaidi H, Sgouros G (2003). Therapeutic Applications of Monte Carlo Calculations in Nuclear Medicine. Institute of Physics Publishing, 364 pp. ISBN: 0-7503816-8. 